of the MMN deficit in schizophrenia and its neurological basis.

Method: EEG and functional magnetic resonance imaging (fMRI) data were obtained from first-episode and chronic patients with schizophrenia, and matched controls. A duration-deviant MMN paradigm was used in which participants heard an unattended random series of tones consisting of standards $(92 \%, 50 \mathrm{~ms})$ and deviants $(8 \%, 100 \mathrm{~ms})$. High-resolution MMNs were obtained as the difference between deviant and standard ERPs. Cortically constrained LORETA current source density analysis was performed using realistic head models.

Results: A reduction in MMN amplitude was seen in younger patients ( 40 years). This is consistent with our previous findings. Current source density analysis of the early phase of the MMN suggests that the major cortical generator of the MMN lies in the superior temporal gyrus (STG) as expected. The latter phase of the MMN engages more anterior cortical regions including premotor cortex. Patients show reduced activity in STG but increased activity in right premotor cortex. fMRI analysis showed that patients have greater activation in the insula and premotor cortex, whereas controls show greater activation in middle frontal gyrus.

Conclusions: The results are consistent with a deficit that onsets early in the disorder, that is associated with substantially reduced processing within auditory cortex and that leads to different patterns of activation in frontal cortical regions in patients compared with controls.

\section{Investigation of circadian disruption in bipolar disorder}

\author{
J Fullerton', A Heath', P MitchelI², P Schofield \\ Prince of Wales Medical Research Institute; and 'Mood Disorders Unit, \\ The Black Dog Institute, Sydney, Australia
}

Background: There is a growing body of evidence implicating circadian disruption as a possible mechanism underlying the pathogenesis of bipolar disorder. The circadian clock is entrained by changes in our external environment and regulates many of our bodies functions, including our sleep-wake cycle, metabolism, thermoregulation and blood pressure. While the measurement of circadian rhythmicity in mice is relatively straight forward through automated recording of home cage activity, the measurement of circadian function/ disruption in humans is more difficult and costly, requiring each patient to undergo actigraphy in a sleep laboratory over a period of several days.

Methods: To collect data on a scale sufficient for genetic studies, a more efficient method of monitoring circadian function in humans is required to determine whether circadian disruption is involved in the primary pathogenesis of bipolar disorder or whether symptomatic sleep disruption is a secondary defect. We are investigating the role of circadian disruption in bipolar disorder, by genetic association, and rhythmic gene expression using immortalized B lymphocytes from a cohort of Australian bipolar families.

Results: Circadian timing in immortalized B lymphocytes is synchronized by serum shock, and circadian gene expression ensues for at least $56 \mathrm{~h}$ post synchronization.

Conclusions: Peripheral tissues, including immortalized blood cells, can be used as a cheap and higher throughput method to measure circadian output in humans and will be a useful adjunct to elucidating the molecular mechanisms underlying bipolar disorder.

\section{Silence is golden? Improving cognitive performance in schizophrenia}

\section{Galletly', M Proeve ${ }^{2}$, C Field ${ }^{2}$, K Harvey ${ }^{2}$}

University of Adelaide; and 2University of South Australia, Adelaide, Australia

Cognitive impairment is a common and disabling symptom of schizophrenia and has adverse effects on functional outcome. Improvement in cognitive function has now been identified as a major target in the treatment of schizophrenia. Cognitive remediation strategies where people with schizophrenia are required to speak aloud while performing learning tasks have shown some success. The present study compared performance on tasks requiring parallel vocalization with task performance when the person did not speak aloud. Results showed selective benefits of verbal strategy on a neuropsychological task requiring multiple executive functions but not on tasks requiring simpler single-component cognitive functions.

\section{Can consent be uninformed? Suggested reform of sexual offences against persons with mental impairment}

\section{Graydon}

Medicine, Nursing and Health Sciences, Murdoch University, WA, Australia

In R v Morgan (1970), the Supreme Court of Victoria stated that for incapacity to consent to be proved, it must be shown that a person 'has not sufficient knowledge or understanding to comprehend (a) that what is 